\title{
Transpterygoid Approach to a Dermoid Cyst in Pterygopalatine Fossa
}

\author{
Alexandre Beraldo Ordones ${ }^{1}$ Marco Aurélio Fornazieri ${ }^{1}$ Fábio de Rezende Pinna ${ }^{1}$ \\ Thiago Freire Pinto Bezerra ${ }^{1}$ Richard Louis Voegels ${ }^{1}$ Luiz Ubirajara Sennes ${ }^{1}$ \\ ${ }^{1}$ Department of Otolaryngology, The School of Medicine, The \\ Address for correspondence Marco Aurélio Fornazieri, MD, Dr. Eneas \\ University of São Paulo, São Paulo/SP, Brazil \\ de Carvalho Aguiar Street, 255/6th floor-6167, Cerqueira Cesar/SP, \\ Brazil (e-mail: marcofornazieri@gmail.com).
}

Int Arch Otorhinolaryngol 2014;18:83-86.

\begin{abstract}
Objective To describe a case of dermoid cyst arising from the pterygopalatine fossa and review the literature.

Methods We report a case of a 23-year-old man who suffered a car accident 2 years before otolaryngologic attendance. He had one episode of generalized tonic-clonic seizure and developed a reduction of visual acuity of the left side after the accident. Neurologic investigation was performed and magnetic resonance imaging revealed an incidental finding of a heterogeneous ovoid lesion in the pterygopalatine fossa, hyperintense on T2-weighted imaging.

Results Endoscopic sinus surgery with transpterygoid approach was performed. The ovoid lesion was noted in the pterygopalatine fossa. Puncture for intraoperative evaluation showed a transparent thick fluid. Surprisingly, hair and sebaceous glands were found inside the cyst capsule. The cyst was excised completely. Histologic

Keywords

- dermoid cyst

- epidermal cyst

- head and neck neoplasms

- skull base

- nasal cavity

- pterygopalatine fossa examination revealed a dermoid cyst. The patient currently has no evidence of recurrence at 1 year postoperatively.

Conclusion This unique case is a rare report of a dermoid cyst incidentally diagnosed. An endoscopic transnasal transpterygoid approach may be performed to treat successfully this kind of lesion. Although rare, it should be considered in the differential diagnosis of expansive lesions in the pterygopalatine fossa, including schwannoma, angiofibroma, esthesioneuroblastoma, osteochondroma, cholesterol granuloma, hemangioma, lymphoma, and osteoma.
\end{abstract}

\section{Introduction}

Dermoid cysts are congenital defects involving pluripotent stem cells arising from an ectopic site. This disease presents a slightly higher predominance in males, and most patients are diagnosed within the first fourth decades of life. ${ }^{1}$ Although up to one third of dermoid cysts are found at birth, most of them are diagnosed in the second and third decades. ${ }^{2}$

Almost $7 \%$ of all dermoids are located in the head and neck. ${ }^{3}$ They are frequently found in the region of the lateral part of the eyebrow, in the periorbital region, and in the midline nasal region. In the neck they usually occur in the submental region, above the hyoid and always in the midline. ${ }^{3}$ This tumor accounts for fewer than $5 \%$ of all intracranial masses. ${ }^{2}$

Tumors arising from the sinonasal region usually present late as their symptoms are often banal and may be overlooked by patients and their clinicians. The recent onset of unilateral nasal symptoms, without improvement with medical therapy, and orbital and neurologic symptoms should be investigated with imaging studies. Computed tomography (CT) and magnetic resonance imaging (MRI) are used to characterize tumors of this region. ${ }^{4}$

Endoscopic sinus surgery is a well-established technique for the treatment of sinus diseases, including chronic sinusitis received

May 15, 2013

accepted

June 27, 2013
DOI http://dx.doi.org/

10.1055/s-0033-1353370. ISSN 1809-9777.
Copyright ( $\odot 2014$ by Thieme Publicações License terms Ltda, Rio de Janeiro, Brazil

() (1) $\odot \circledast$ 


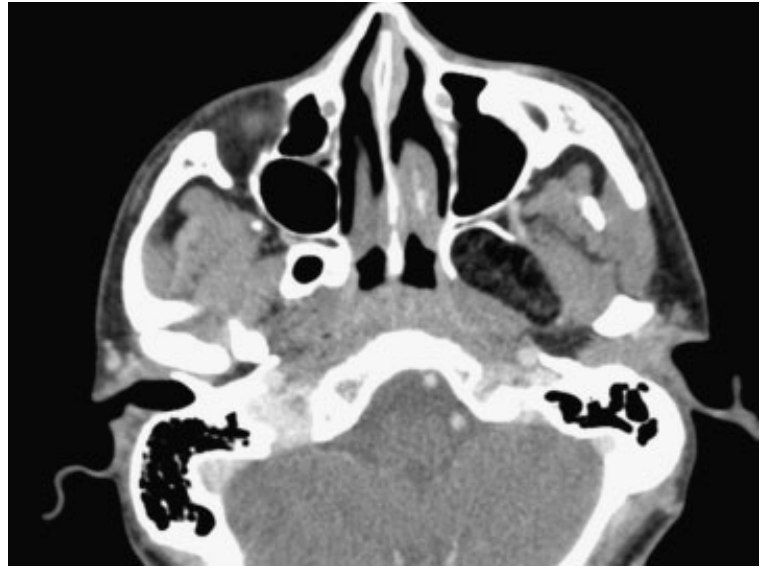

Fig. 1 Computed tomography showing a heterogeneous lobulated lesion in the infratemporal region.

and nasal neoplasms. Endoscopic sinus surgery to approach nasal neoplasms is limited, as the tumor can be too extensive. ${ }^{5}$ In this report, we discuss diagnosis, differential diagnosis, and possible approaches to lesions arising from the pterygopalatine fossa.

\section{Case Report}

A 23-year-old man was admitted to a tertiary care center after a car accident, in which he sustained a pelvic/femoral fracture. There was no evidence of any associated injury, and a conversion to tracheostomy after long-term intubation was performed. He also presented a first episode of generalized tonic-clonic seizure. In addition, he presented reduced visual acuity in the left side.

The patient was referred for brain MRI; a heterogeneous ovoid lesion hyperintense in T2-weighted imaging in the pterygopalatine fossa was discovered. As a matter of fact, this lesion was indenting the middle fossa and was closely related to the maxillary artery, sphenopalatine ganglia, and maxillary posterior wall. The patient was referred to the
Otolaryngology Department of Clinics Hospital-University of São Paulo.

Prior to surgery, a wide range of differential cell counts were obtained. Laboratory workup, including a complete blood count with differentials and determination of liver enzymes and electrolytes, showed no abnormal findings. Brain CT showed a heterogeneous lobulated lesion in the left masticator space (infratemporal region), $3 \times 3,5 \times 2 \mathrm{~cm}$ wide, medial to lateral pterygoid muscle, with erosion of the inferior wall of foramen rotundum (-Fig. 1). We repeated brain MRI, which showed a lobulated extra-axial formation in the left masticator space, between lateral and medial pterygoid muscles, with fat heterogeneous tissue, hypointense in T2-weighted image, gadolinium-enhanced in T1-weighted image (-Fig. 2). There were small hyperintense lesions in T1-weighted images in the suprasellar cistern and in the sylvian fissure, suggesting previous dermoid rupture (-Fig. 2B).

A transpterygoid transnasal endoscopic approach and resection of the lesion was performed. The puncture of the lesion showed initially a transparent thick liquid. Unexpectedly, hair and sebaceous glands were found inside the cyst capsule (-Fig. 3). The cyst was excised completely.

Histopathologic examination confirmed the diagnosis of a dermoid cyst. The patient's postoperative course was unremarkable.

\section{Discussion}

Benign and malignant tumors can arise from any of the structures within the infratemporal fossa and parapharyngeal space. ${ }^{6}$ Dermoid inclusion cysts are benign tumors and are mainly unilocular and expand slowly, enlarging over years or decades, by the accumulation of cutaneous products. They may show lipid content, derived from sebaceous secretions, and secretions of apocrine sweat glands and hair. ${ }^{2}$

The causes of dermoids include failure of surface ectoderm to separate from underlying structures and sequestration of

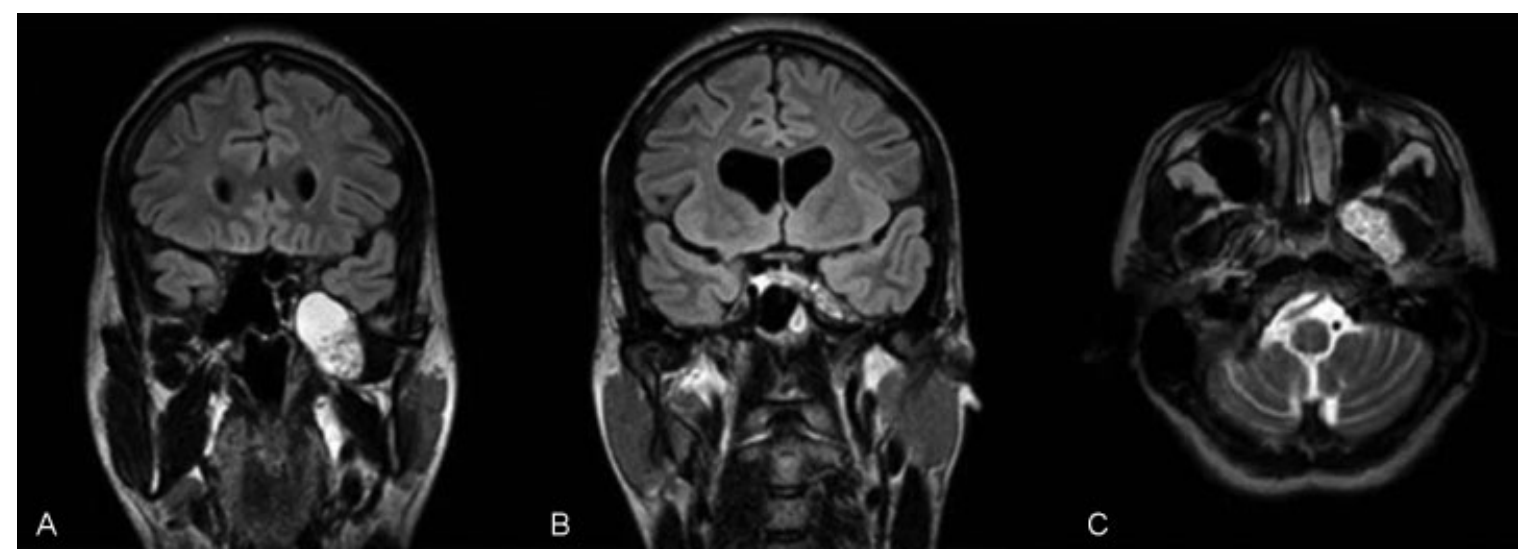

Fig. 2 Magnetic resonance imaging (MRI) of the lesion with heterogenous content in the inferior region. (A, B) T1-weighted coronal MRI. (C) T1-weighted axial MRI showing invasion of the infratemporal fossa. 


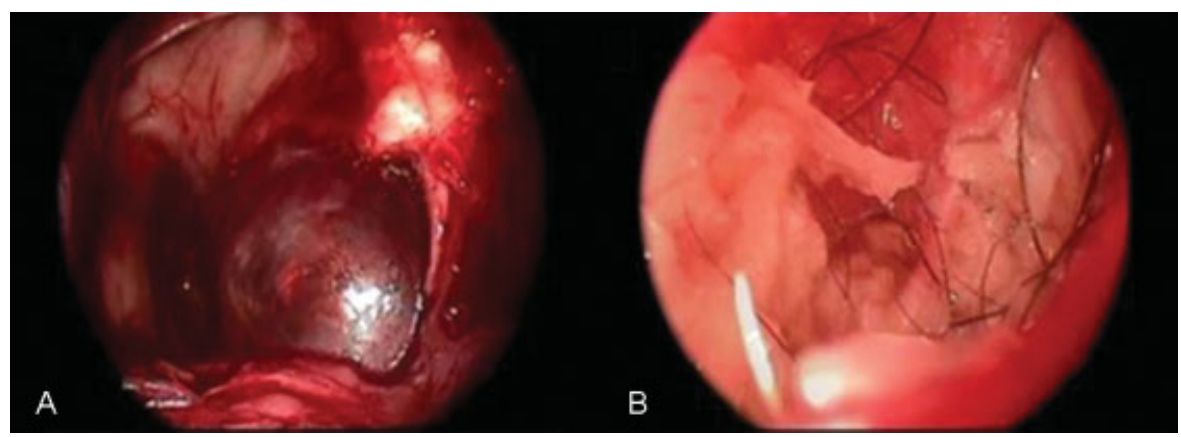

Fig. 3 Intraoperative endoscopic visualization of the dermoid cyst.

surface ectoderm at lines of epithelial fusion during embryonic development. Most congenital dermoid cysts probably arise due to an embryologic accident during early stages of development, between the third and fifth weeks of gestation. ${ }^{2,3}$

Clinical examination of the fossa pterygoid is difficult because it is deep lying and not easily accessible. Lesions are often discovered only at a late stage either because there are no clinical signs or, if they do occur, they are so common as to be overlooked. ${ }^{1,6,7}$ In our case, the diagnosis was incidental. Tumors that arise in pterygopalatine fossa are usually asymptomatic. CT and MRI provide precise imaging information and may be necessary for making the diagnosis. In addition, imaging data can help to differentiate infection from tumor lesions and primary tumors from secondary tumors. ${ }^{1,7} \mathrm{Al}-$ though they are slow-growing, dermoid inclusions cysts produce pressure changes on surrounding structures that are visible at radiograph study.

In a case series, Yu et al evaluated 86 patients with tumoral lesions of pterygopalatine and infratemporal spaces. ${ }^{1}$ Most of the lesions (81\%) originated in oral and maxillofacial regions other than pterygopalatine and infratemporal spaces and extended to this region. Only one case of teratoma was found. The most frequent diseases reported among the other cases were squamous cells carcinoma, adenoid cystic carcinoma, inflammatory disease, and hemangioma.

Differential diagnosis in cases of tumoral lesions in pterygoid fossa should be performed ( - Table 1 ). ${ }^{1,8-13}$

Treatment of dermoid cysts consists of complete surgical excision of the lesion, avoiding recurrence. ${ }^{6}$ Rupture of dermoid cysts can produce severe chemical meningitis, usually attributed to the irritating effects of the cholesterol in the cellular debris. $^{2}$ In our case, brain MRI showed signs of previous rupture, which may account for the patient's epilepsy.

Uppal et al excised a dermoid cyst in the infratemporal fossa, extending inferiorly to the parapharyngeal space, using a lateral approach to that region. ${ }^{6}$ In our case, we performed a minimally invasive treatment, using an endoscopic transnasal transpterygoid approach with a wide access to the lesion. It was possible to proceed with complete excision of the tumor, without intraoperative complications.
The clinical diagnosis and management of these lesions can be challenging because of the relative inaccessibility of the region, which contains the maxillary artery, the maxillary nerve, and the pterygopalatine ganglia, with its branches.

Surgical advantages of the endoscopic endonasal approach in comparison with traditional transcranial approaches include a more direct midline exposure, decreased brain parenchyma injury, and lack of neurovascular structure manipulation. From the patient's perspective, decreased surgery time, decreased length of stay, increased patient comfort, and lack of external incision are advantages of the endoscopic endonasal approach.

Although rare, dermoid cysts should be considered in the differential diagnosis of expansive lesions in the pterygopalatine fossa and can be excised by a transnasal transpterygoid endoscopic approach.

Table 1 Differential diagnosis of lesions in pterygoid region

\begin{tabular}{|l|l|}
\hline Meningoencephalocele & Malignant lymphoma \\
\hline Maxillary sinus carcinoma & Squamous cell carcinoma \\
\hline Olfactory neuroblastoma & Inflammatory disease \\
\hline Osteochondroma & Adenoid cystic carcinoma \\
\hline Cholesterol granuloma & Adenocarcinoma \\
\hline Myoepithelial carcinoma & Hemangioma \\
\hline Ameloblastoma & Leiomyosarcoma \\
\hline Malignant fibrohistiocytoma & Undifferentiated carcinoma \\
\hline Giant-cell tumor & Chondrosarcoma \\
\hline Keratocyst & Osteoblastoma \\
\hline Mixed tumor & Malignant myoepithelioma \\
\hline Mucoepidermoid carcinoma & Liposarcoma \\
\hline Hemangiopericytoma & Hemangioendothelioma \\
\hline Undifferentiated sarcoma & Malignant mixed tumor \\
\hline Lymphangioma & Neurofibroma \\
\hline Rhabdomyosarcoma & \\
\hline
\end{tabular}




\section{Author Contributions}

All authors participated in the medical treatment of the patient, including postoperative care. In addition, they performed data collection and analysis and the writing of the paper.

\section{References}

1 Yu Q, Wang P, Shi H, Luo J, Sun D. The lesions of the pterygopalatine and infratemporal spaces: computed tomography evaluation. Oral Surg Oral Med Oral Pathol Oral Radiol Endod 1998;85:742-751

2 Smirniotopoulos JG, Chiechi MV. Teratomas, dermoids, and epidermoids of the head and neck. Radiographics 1995;15:1437-1455

3 Vrabec JT, Schwaber MK. Dermoid tumor of the middle ear: case report and literature review. Am J Otol 1992;13:580-581

4 European Position Paper on Endoscopic Management of Tumors of the Nose, Paranasal Sinuses and Skull Base. Rhinology 2010 (Suppl 22):1-143

5 Snyderman CH, Pant H, Carrau RL, Prevedello D, Gardner P, Kassam $\mathrm{AB}$. What are the limits of endoscopic sinus surgery? The expanded endonasal approach to the skull base. Keio J Med 2009;58: $152-160$

6 Uppal HS, D'Souza AR, De R, Irving RM. Dermoid cyst of the infratemporal fossa. J Laryngol Otol 2002;116:150-152

7 Faye N, Lafitte F, Williams M, et al. The masticator space: from anatomy to pathology. J Neuroradiol 2009;36:121-130

8 Nishikawa T, Ishida H, Nibu K. A rare spontaneous temporal meningoencephalocele with dehiscence into the pterygoid fossa. Auris Nasus Larynx 2004;31:429-431

9 Souza RP, et al. Maxillary sinus carcinoma: an analysis of ten cases. Radiol Bras 2006;39:397-400

10 Seccia V, Lenzi R, Casani AP, Muscatello L. Ectopic olfactory neuroblastoma arising in the pterygopalatine fossa. Otolaryngol Head Neck Surg 2010;142:460-461

$11 \mathrm{Wu}$ W, Hu X, Lei D. Giant osteochondroma derived from pterygoid process of sphenoid. Int J Oral Maxillofac Surg 2007;36:959-962

12 Weiland DA, Aygun N. An unusual presentation of a cholesterol granuloma in a pneumatized pterygoid process of the sphenoid sinus. Otolaryngol Head Neck Surg 2007;136:153-154

13 Ghosh A, Saha S, Saha VP, Sadhu A, Chattopadhyay S. Infratemporal fossa myoepithelial carcinoma-a rare case report. Oral Maxillofac Surg 2009;13:59-62 